\title{
GREEN'S FUNCTION FOR IMPULSIVE PERIODIC SOLUTIONS IN ALTERNATELY ADVANCED AND DELAYED DIFFERENTIAL SYSTEMS AND APPLICATIONS
}

\author{
Kuo-Shou CHIU
}

Departamento de Matemática, Facultad de Ciencias Básicas, Universidad Metropolitana de Ciencias de la Educación, José Pedro Alessandri 774, Santiago, CHILE

\begin{abstract}
In this paper we investigate the existence of the periodic solutions of a nonlinear impulsive differential system with piecewise alternately advanced and retarded arguments, in short IDEPCAG, that is, the argument is a general step function. We consider the critical case, when associated linear homogeneous system admits nontrivial periodic solutions. Criteria of existence of periodic solutions of such system are obtained. In the process we use the Green's function for impulsive periodic solutions and convert the given the IDEPCAG into an equivalent integral equation system. Then we construct appropriate mappings and employ Krasnoselskii's fixed point theorem to show the existence of a periodic solution of this type of nonlinear impulsive differential systems. We also use the contraction mapping principle to show the existence of a unique impulsive periodic solution. Appropriate examples are given to show the feasibility of our results.
\end{abstract}

\section{INTRODUCTION}

Among the functional differential equations, Myshkis 27 proposed to study differential equations with piecewise constant arguments: DEPCA. The theory of scalar DEPCA of the type

$$
\frac{d x(t)}{d t}=f(t, x(t), x(\beta(t))), \beta(t)=[t] \text { or } \beta(t)=2\left[\frac{t+1}{2}\right],
$$

where [.] signifies the greatest integer function, was initiated in [8.33] and has been developed by many authors $6,20,28,35,36,38$. DEPCA are hybrid equations,

2020 Mathematics Subject Classification. Primary 34A37, 34K13; Secondary 34A38, 34B27, $37 \mathrm{C} 25$.

Keywords and phrases. Impulsive differential equation, piecewise constant arguments of generalized type, Green's function, periodic solutions, fixed point theorems.

\kschiu@umce.cl

(D) 0000-0002-3823-5898. 
because they combine the characteristics of continuous systems and discrete equations. The continuity of a solution in points that unite two consecutive intervals implies the existence of recursive relations for the solution in such points. The equations are thus similar in structure to those found in certain "sequentially continuous" models of the dynamic ones of the disease of the treaty by S. Busenberg and K. L. Cooke [5]. Others applications of DEPCA are discussed in 35].

These kinds of systems can usually be formulated as first or second order differential equations with piecewise constant arguments. Examples in practice include machinery driven by servo units, charged particles moving in a piecewise constantly varying electric field, and elastic systems impelled by a Geneva wheel.

Impulsive differential equations arise naturally in the description of physical and biological phenomena that are subjected to instantaneous changes at some time instants called moments. For a good account on this theory, which has seen a significant development over the past decades we refer the interested reader to the monographs 26,31] and the references therein.

There are many papers that study the qualitative behaviours of the impulsive differential equations $22,25,37$. Among these investigations stability and instability problems are very interesting. Impulses can make unstable systems stable and stable systems can become unstable after impulse effects 2,24 .

To the best of our knowledge, there are only a few papers involving impulsive differential equations with piecewise constant arguments $3,12,16,21,23,37$.

In 2000, Wiener and Lakshmikantham 37] proved the existence and uniqueness of solutions of the initial value problem

$$
x^{\prime}(t)=f(t, x(t), x(g(t))), x(0)=x_{0},
$$

and they gave some oscillation and stability results for the same problem, where $f$ is a continuous function and $g:[0, \infty) \rightarrow[0, \infty), g(t) \leq t$ is a step function.

In 2009, F. Karakoc et al. 21 consider the first order linear scalar impulsive delay differential equation of the type

$$
\begin{aligned}
& y^{\prime}(t)+a(t) y(t)+b(t) y([t-1])=0, t \neq n, \\
& \Delta y(n)=d_{n} y(n), n \in \mathbb{N},
\end{aligned}
$$

and they also studied the existence of oscillatory and periodic solutions, where $a, b: \mathbb{R}^{+} \rightarrow \mathbb{R}$ continuous functions and $d_{n} \in \mathbb{R} \backslash\{1\}, \Delta y(n)=y\left(n^{+}\right)-y\left(n^{-}\right)$, $y\left(n^{+}\right)=\lim _{t \rightarrow n^{+}} y(t)$ and $y\left(n^{-}\right)=\lim _{t \rightarrow n^{-}} y(t)$.

In 2017, Gizem S. Oztepe et al. $[29$ consider the second order impulsive differential equation with a piecewise constant argument of the type

$$
\begin{aligned}
x^{\prime \prime}(t)-a^{2} x(t) & =b x([t]), t \neq n \in \mathbb{Z}^{+}, \quad t \geq 0, \\
\Delta x^{\prime}(n) & =d x^{\prime}(n), \quad n \in \mathbb{Z}^{+},
\end{aligned}
$$

and they gave some oscillatory and periodic results, where $a, b, d \in \mathbb{R} \backslash\{0\}$. 
In 2019, R. Torres et al. 7 consider the impulsive differential equation with piecewise constant argument of generalized type (IDEPCA of generalized type) and they gave certain conditions for the existence of an asymptotic equilibrium of non-linear and semi-linear IDEPCA systems of generalized type.

Recently, Chiu [19], using Green's function and fixed point theory, obtained some sufficient conditions for the existence and uniqueness of periodic (or harmonic) and subharmonic solutions of quasilinear DEPCA systems of generalized type.

Let $\mathbb{Z}, \mathbb{N}$ and $\mathbb{R}$ be the set of all integers, natural, real and complex numbers, respectively. Fix two real sequences $t_{i}, \gamma_{i}, i \in \mathbb{Z}$, such that $t_{i}<t_{i+1}, t_{i} \leq \gamma_{i} \leq t_{i+1}$ for all $i \in \mathbb{Z}, t_{i} \rightarrow \pm \infty$ as $i \rightarrow \pm \infty$. Let $\gamma: \mathbb{R} \rightarrow \mathbb{R}$ be a step function given by $\gamma(t)=\gamma_{i}$ for $t \in I_{i}=\left[t_{i}, t_{i+1}\right)$ and consider the DEPCA (1) with this general $\gamma$. In this case we speak of DEPCA of generalized type, in short DEPCAG. Theory and practice of DEPCAG have been discussed extensively in [1,9 11, 13, 14, 17, 18,30 and IDEPCA of generalized type have been discussed in $7 . \mid 15]$.

The main purpose of this paper is to establish some simple criteria for the existence of periodic solutions of a nonlinear system of impulsive differential systems with alternately of advanced and retarded arguments of generalized type (in short IDEPCAG):

$$
\begin{aligned}
& z^{\prime}(t)=A(t) z(t)+f(t, z(t), z(\gamma(t)))+g(t, z(t), z(\gamma(t))), \quad t \neq t_{k}, \\
& \left.\Delta z\right|_{t_{k}}=J_{k}\left(z\left(t_{k}^{-}\right)\right), \quad k \in \mathbb{Z},
\end{aligned}
$$

where $t \in \mathbb{R}, A: \mathbb{R} \rightarrow \mathbb{R}^{n \times n}, f: \mathbb{R} \times \mathbb{R}^{n} \times \mathbb{R}^{n} \rightarrow \mathbb{R}^{n}$ and $g: \mathbb{R} \times \mathbb{R}^{n} \times \mathbb{R}^{n} \rightarrow \mathbb{R}^{n}$ and $J_{k}: \mathbb{R}^{n} \rightarrow \mathbb{R}^{n}$ are continuous in their respective arguments. Moreover, $\Delta z\left(t_{k}\right)$ denotes $z\left(t_{k}\right)-z\left(t_{k}^{-}\right)$, where $z\left(t_{k}^{-}\right)=\lim _{h \rightarrow 0^{-}} z\left(t_{k}+h\right)$.

In the analysis, we use the idea of the Green's function for impulsive periodic solutions and convert the IDEPCAG system 2a -2 (2b) into an equivalent integral equation. Then we employ Krasnoselskii's fixed point theorem and show the existence of an impulsive periodic solution of the IDEPCAG system (2a)-(2b) in Theorem 13. We also obtain the existence of a unique periodic solution in Theorem 15 employing the contraction mapping principle as the basic mathematical tool. Furthermore, appropriate examples are given to show the feasibility of our results. For example, some new and interesting sufficient conditions are obtained to guarantee the existence of the periodic solutions of nonlinear integro-differential equations with the IDEPCAG system

$$
\begin{aligned}
z^{\prime}(t) & =A(t) z(t)+\int_{-\infty}^{t} C(t, s, z(\gamma(s))) d s+g(t, z(t), z(\gamma(t))), \quad t \neq t_{k}, \\
\left.\Delta z\right|_{t_{k}} & =J_{k}\left(z\left(t_{k}^{-}\right)\right), \quad k \in \mathbb{Z} .
\end{aligned}
$$

In our paper we assume that the solutions of the IDEPCAG system (2a)-(2b) are continuous functions with possible points of discontinuity of the first kind at $t_{k}, k \in \mathbb{Z}$. But the deviating argument $\gamma(t)$ is discontinuous. Thus, in general, the right-hand side of the IDEPCAG system (2a)-(2b) has discontinuities at moments 
$t_{k} \in \mathbb{R}, k \in \mathbb{Z}$. As a result, we consider the solutions of the IDEPCAG system as functions, which are continuous and continuously differentiable within intervals $\left[t_{k}, t_{k+1}\right), k \in \mathbb{Z}$.

We use the following definition, which is a version of a definition from [12 16, modified for our general case.

Definition 1. A function $z$ is a solution of the IDEPCAG system (2a)-(2b) in $\mathbb{R}$ if

i) $z(t)$ is continuous for $t \in \mathbb{R}$ with the possible exception of the points $t=t_{k}$, $k \in \mathbb{Z}$.

ii) $z(t)$ is right continuous and has left-hand limits at the points $t=t_{k}, k \in \mathbb{Z}$.

iii) $z(t)$ is differentiable and satisfies (2a) for any $t \in \mathbb{R}$ with the possible exception of the points $t=t_{k}, k \in \mathbb{Z}$, where one-sided derivatives exist.

iv) $z\left(t_{k}\right)$ satisfies the impulsive effects (2b), $k \in \mathbb{Z}$.

The rest of the paper is organized as follows. In the next section, we introduce and show some properties about the Green's function. Section 3 is devoted to establishing some criteria for the existence and uniqueness of impulsive periodic solutions of the IDEPCAG system (2a)-(2b). Green's operator and Banach, Schauder and Krasnoselskii's fixed point theorem below are fundamental to obtain the main results. Furthermore, appropriate examples are provided in the last section to show the feasibility of our results.

\section{GREen's FUNCTION AND PERIODICITY}

In this section we state and define the Green's function for periodic solutions in impulsive alternately advanced and delayed differential systems $2 \mathrm{a}-2 \mathrm{2b})$.

Let $I$ be the $n \times n$ identity matrix. Denote by $\Phi(t, s), \Phi(s, s)=I, t, s \in \mathbb{R}$, the fundamental matrix of solutions of the homogeneous system (4).

From now on the following assumption will be needed:

$\left(\mathcal{N}_{\omega}\right)$ The homogeneous equation

$$
y^{\prime}(t)=A(t) y(t)
$$

does not admit any nontrivial $\omega$-periodic solution.

Remark 2. For $\tau \in \mathbb{R}$, the condition $\left(\mathcal{N}_{\omega}\right)$ is equivalent to the matrix $(I-\Phi(\tau+\omega, \tau))$ is non-singular.

Definition 3. Suppose that the condition $\left(\mathcal{N}_{\omega}\right)$ holds. For each $t, s \in[\tau, \tau+\omega]$, the Green's function for the IDEPCAG system (2a)-(2b) is given by

$$
G(t, s)= \begin{cases}\Phi(t)(I+D) \Phi^{-1}(s), & \tau \leq s \leq t \leq \tau+\omega \\ \Phi(t) D \Phi^{-1}(s), & \tau \leq t<s \leq \tau+\omega\end{cases}
$$

where, $\Phi(t)$ is a fundamental solution of (4) and

$$
D=\left(\left(\Phi^{-1}(\tau) \Phi(\tau+\omega)\right)^{-1}-I\right)^{-1} .
$$


We note that the condition $\left(\mathcal{N}_{\omega}\right)$ implies the existence of the matrix $D$.

Remark 4. In the case of $A(t)=\operatorname{diag}\left\{a_{i}(t)\right\}_{i \in\{1, \ldots, n\}}$, the Green's function $G(t, s)=$ $\operatorname{diag}\left\{G_{i}(t, s)\right\}_{i \in\{1, \ldots, n\}}$ of the IDEPCAG system (2a)-2b) has the form

$$
G_{i}(t, s)=\left\{\begin{array}{l}
\left(\frac{\exp \left(\int_{\tau}^{\tau+\omega} a_{i}(u) d u\right)}{\exp \left(\int_{\tau}^{\tau+\omega} a_{i}(u) d u\right)-1}\right) \exp \left(\int_{t}^{s} a_{i}(\kappa) d \kappa\right), \tau \leq s \leq t \leq \tau+\omega, \\
\left.\frac{1}{\exp \left(\int_{\tau}^{\tau+\omega} a_{i}(u) d u\right)-1}\right) \exp \left(\int_{t}^{s} a_{i}(\kappa) d \kappa\right), \tau \leq t<s \leq \tau+\omega .
\end{array}\right.
$$

Lemma 5. Suppose that the condition $\left(\mathcal{N}_{\omega}\right)$ holds. Then the Green's function $G(t, s)$ is double $\omega$-periodic, i.e., $G(t+\omega, s+\omega)=G(t, s)$.

Proof of this affirmation was omitted, as it can be demonstrated in the same way as Lemma 2.2 in 19 .

\section{Existence of Periodic Solutions}

In this section, we prove the main theorems of this paper, by using Banach, Schauder and Krasnoselskii's fixed point theorems, we investigate the existence and uniqueness of periodic and subharmonic solutions of the IDEPCAG system, so we recall the IDEPCAG system (2a)-(2b):

$$
\left\{\begin{array}{l}
z^{\prime}(t)=A(t) z(t)+f(t, z(t), z(\gamma(t)))+g(t, z(t), z(\gamma(t))), \quad t \neq t_{k}, \\
\left.\Delta z\right|_{t_{k}}=J_{k}\left(z\left(t_{k}^{-}\right)\right), \quad k \in \mathbb{Z} .
\end{array}\right.
$$

Let $\mathcal{P C}$ be the set of all $n$-vector piecewise continuous function $z(t)$ with points of discontinuity of the first kind at $t=t_{k}, k \in \mathbb{Z}$. For this, a natural Banach space is

$$
\mathcal{P C}_{\omega}=\{z(t) \mid z(t) \in \mathcal{P C}, z(t+\omega)=z(t)\}
$$

with the supremum norm

$$
\|z\|=\sup _{t \in \mathbb{R}}|z(t)|=\sup _{t \in[\tau, \tau+\omega]}|z(t)| .
$$

Consider $f: \mathbb{R} \times \mathbb{R}^{n} \times \mathbb{R}^{n} \rightarrow \mathbb{R}^{n}, g: \mathbb{R} \times \mathbb{R}^{n} \times \mathbb{R}^{n} \rightarrow \mathbb{R}^{n}$ and $J_{k}: \mathbb{R}^{n} \rightarrow \mathbb{R}^{n}$ are continuous functions. For every $t \in \mathbb{R}$, let $i=i(t) \in \mathbb{Z}$ be the unique integer such that $t \in I_{i}=\left[t_{i}, t_{i+1}\right)$. To obtain our main results, we make the following assumptions throughout this paper.

\section{Lipschitz conditions:}

$\left(\mathcal{L}_{f}\right)$ There exist functions $p_{1}, p_{2}: \mathbb{R} \rightarrow[0, \infty)$ such that

$$
\left|f\left(t, x_{1}, y_{1}\right)-f\left(t, x_{2}, y_{2}\right)\right| \leq p_{1}(t)\left|x_{1}-x_{2}\right|+p_{2}(t)\left|y_{1}-y_{2}\right|,
$$


for $t \in \mathbb{R}$ and $x_{1}, y_{1}, x_{2}, y_{2} \in \mathbb{R}^{n}$. Moreover, $\alpha=\sup _{t \in \mathbb{R}}|f(t, 0,0)|$ and

$$
\int_{\tau}^{\tau+\omega}\left[p_{1}(s)+p_{2}(s)\right] d s \leq L_{1}, \omega \in \mathbb{R}_{+}, \tau \in \mathbb{R} .
$$

$\left(\mathcal{L}_{g}\right)$ There exist functions $v_{1}, v_{2}: \mathbb{R} \rightarrow[0, \infty)$ such that

$$
\left|g\left(t, x_{1}, y_{1}\right)-g\left(t, x_{2}, y_{2}\right)\right| \leq v_{1}(t)\left|x_{1}-x_{2}\right|+v_{2}(t)\left|y_{1}-y_{2}\right|,
$$

for $t \in \mathbb{R}$ and $x_{1}, y_{1}, x_{2}, y_{2} \in \mathbb{R}^{n}$. Moreover, $\beta=\sup _{t \in \mathbb{R}}|g(t, 0,0)|$ and

$$
\int_{\tau}^{\tau+\omega}\left[v_{1}(s)+v_{2}(s)\right] d s \leq L_{2}, \omega \in \mathbb{R}_{+}, \tau \in \mathbb{R} .
$$

$\left(\mathcal{L}_{J}\right)$ There exist functions $\wp_{k}: \mathbb{R} \rightarrow[0, \infty), k \in \mathbb{Z}$, such that

$$
\left|J_{k}\left(x_{1}\right)-J_{k}\left(x_{2}\right)\right| \leq \wp_{k}(t)\left|x_{1}-x_{2}\right|,
$$

for $t \in \mathbb{R}$ and $x_{1}, x_{2} \in \mathbb{R}^{n}$. Moreover, $\kappa=\sup _{k \in \mathbb{Z}}\left|J_{k}(0)\right|$ and

$$
\sum_{k=i(\tau)+1}^{i(\tau)+p} \wp_{k}(t) \leq L_{3}, p \in \mathbb{N}, i(\tau) \in \mathbb{Z} .
$$

\section{Periodic conditions:}

$(\mathcal{P})$ There exists $\omega>0$ such that:

1) $A(t), f\left(t, x_{1}, y_{1}\right)$ and $g\left(t, x_{2}, y_{2}\right)$ are periodic functions in $t$ with a period $\omega$ for all $t \geq \tau$.

2) There exists $p \in \mathbb{Z}^{+}$, for which the sequences $\left\{t_{i}\right\}_{i \in \mathbb{Z}},\left\{\gamma_{i}\right\}_{i \in \mathbb{Z}}$, satisfy the $(\omega, p)$ condition, that is

$$
t_{i+p}=t_{i}+\omega, \quad \gamma_{i+p}=\gamma_{i}+\omega, \quad \text { for } \quad i \in \mathbb{Z}
$$

3) The sequence $\left\{J_{i}\right\}_{i \in \mathbb{Z}}$ satisfies

$$
J_{i+p}=J_{i}
$$

some positive integer $p \in \mathbb{N}$.

\section{Invariance conditions:}

$\left(\mathcal{M}_{f}\right)$ For every $\mathcal{R}>0, t \in \mathbb{R},|x|,|y| \leq \mathcal{R}$, there exist functions $m_{1}, m_{2}: \mathbb{R} \rightarrow$ $[0, \infty)$ and positive constants $\rho_{1}, C_{1}$ for which

$$
|f(t, x, y)| \leq m_{1}(t)|x|+m_{2}(t)|y|+\rho_{1},
$$

where $\int_{\tau}^{\tau+\omega}\left[m_{1}(s)+m_{2}(s)\right] d s \leq C_{1}, \omega \in \mathbb{R}_{+}, \tau \in \mathbb{R}$. 
$\left(\mathcal{M}_{g}\right)$ For every $\mathcal{R}>0, t \in \mathbb{R},|x|,|y| \leq \mathcal{R}$, there exist functions $\kappa_{1}, \kappa_{2}: \mathbb{R} \rightarrow$ $[0, \infty)$ and positive constants $\rho_{2}, C_{2}$ for which

$$
|g(t, x, y)| \leq \kappa_{1}(t)|x|+\kappa_{2}(t)|y|+\rho_{2},
$$

where $\int_{\tau}^{\tau+\omega}\left[\kappa_{1}(s)+\kappa_{2}(s)\right] d s \leq C_{2}, \omega \in \mathbb{R}_{+}, \tau \in \mathbb{R}$.

$\left(\mathcal{M}_{J}\right)$ For every $\mathcal{R}>0, t \in \mathbb{R},|x| \leq \mathcal{R}$, there exist functions $\varrho_{k}: \mathbb{R} \rightarrow[0, \infty)$, $k \in \mathbb{Z}$ and positive constants $\rho_{3}, C_{3}$ for which

$$
\left|J_{k}(x)\right| \leq \varrho_{k}(t)|x|+\rho_{3}
$$

where $\sum_{k=i(\tau)+1}^{i(\tau)+p} \varrho_{k}(t) \leq C_{3}, p \in \mathbb{N}, i(\tau) \in \mathbb{Z}$.

Remark 6. Note that $(\omega, p)$ condition is a discrete relation, which moves the interval $I_{i}$ into $I_{i+p}$. Then we have the following consequences:

(i) For any $\tau \in \mathbb{R}$, the interval $[\tau, \tau+\omega]$ can be decomposed as follows:

$$
\left[\tau, t_{i(\tau)+1}\right] \cup \bigcup_{j=i(\tau)+1}^{i(\tau)+p-1} I_{j} \cup\left[t_{i(\tau)+p}, \tau+\omega\right] .
$$

(ii) For $t \in\left[t_{i}, t_{i+1}\right)$, we have

$$
\text { a) } t+\omega \in\left[t_{i+p}, t_{i+p+1}\right), \quad \text { b) } \gamma(t)+\omega \in\left[t_{i+p}, t_{i+p+1}\right) \text {. }
$$

Then

$$
\gamma(t+\omega)=\gamma_{i(t+\omega)}=\gamma_{i(t)}+\omega=\gamma(t)+\omega .
$$

Using Definition 3, Remark 6 and $\omega$-periodicity of the Green's function, we have obtained the following Proposition.

Proposition 7. Suppose that the conditions $\left(N_{\omega}\right)$ and $(P)$ hold. Let $(\tau, z(\tau)) \in$ $\mathbb{R} \times \mathbb{R}^{n}$. Then, $z(t)=z(t, \tau, z(\tau))$ is an $\omega$-periodic solution on $\mathbb{R}$ of the IDEPCAG system 2a-2b if and only if $z(t)$ is an $\omega$-periodic solution of the integral equation system

$$
\begin{aligned}
z(t)=\int_{\tau}^{\tau+\omega} & G(t, s)[f(s, z(s), z(\gamma(s)))+g(s, z(s), z(\gamma(s)))] d s \\
& +\sum_{k=i(\tau)+1}^{i(\tau)+p} G\left(t, t_{k}\right) J_{k}\left(z\left(t_{k}^{-}\right)\right)
\end{aligned}
$$

where the Green's function $G(t, s)$ is defined by (5). 
Proof. If $z(t)$ is an $\omega$-periodic solution of the integral equation system (16), let $t \neq t_{k}^{-}$, then we have

$$
\begin{aligned}
& \quad \frac{d}{d t}\left(\int_{\tau}^{\tau+\omega} G(t, s)[f(s, z(s), z(\gamma(s)))+g(s, z(s), z(\gamma(s)))] d s\right) \\
& =\Phi^{\prime}(t) \int_{\tau}^{t}(I+D) \Phi^{-1}(s)[f(s, z(s), z(\gamma(s)))+g(s, z(s), z(\gamma(s)))] d s \\
& \quad+\Phi(t)(I+D) \Phi^{-1}(t)[f(s, z(s), z(\gamma(s)))+g(s, z(s), z(\gamma(s)))] \\
& \quad+\Phi^{\prime}(t) \int_{t}^{\tau+\omega}\left\{D \Phi^{-1}(s)[f(s, z(s), z(\gamma(s)))+g(s, z(s), z(\gamma(s)))]\right\} d s \\
& \quad-\Phi(t) D \Phi^{-1}(t)[f(t, z(t), z(\gamma(t)))+g(t, z(t), z(\gamma(t)))] \\
& =A(t)\left(\int_{\tau}^{\tau+\omega} G(t, s)[f(s, z(s), z(\gamma(s)))+g(s, z(s), z(\gamma(s)))] d s\right) \\
& \quad+[f(t, z(t), z(\gamma(t)))+g(t, z(t), z(\gamma(t)))] .
\end{aligned}
$$

Similarly,

$$
\frac{d}{d t}\left(\sum_{k=i(\tau)+1}^{i(\tau)+p} G\left(t, t_{k}\right) J_{k}\left(z\left(t_{k}^{-}\right)\right)\right)=A(t)\left(\sum_{k=i(\tau)+1}^{i(\tau)+p} G\left(t, t_{k}\right) J_{k}\left(z\left(t_{k}^{-}\right)\right)\right) .
$$

Hence

$$
z^{\prime}(t)=A(t) z(t)+f(t, z(t), z(\gamma(t)))+g(t, z(t), z(\gamma(t))), \quad t \neq t_{k} .
$$

For any $t=t_{j}, j \in\{i(\tau)+1, \ldots, i(\tau)+p\}$, we have from 16 that

$$
\begin{aligned}
z\left(t_{j}\right)=\int_{\tau}^{\tau+\omega} & G\left(t_{j}, s\right)[f(s, z(s), z(\gamma(s)))+g(s, z(s), z(\gamma(s)))] d s \\
& +\sum_{k=i(\tau)+1}^{i(\tau)+p} G\left(t_{j}, t_{k}\right) J_{k}\left(z\left(t_{k}^{-}\right)\right)
\end{aligned}
$$

and

$$
\begin{aligned}
z\left(t_{j}^{-}\right)=\int_{\tau}^{\tau+\omega} & G\left(t_{j}^{-}, s\right)[f(s, z(s), z(\gamma(s)))+g(s, z(s), z(\gamma(s)))] d s \\
& +\sum_{k=i(\tau)+1}^{i(\tau)+p} G\left(t_{j}^{-}, t_{k}\right) J_{k}\left(z\left(t_{k}^{-}\right)\right) .
\end{aligned}
$$


Therefore

$$
\begin{aligned}
\left.\Delta z\right|_{t_{j}}= & z\left(t_{j}\right)-z\left(t_{j}^{-}\right) \\
= & \int_{\tau}^{\tau+\omega}\left(G\left(t_{j}, s\right)-G\left(t_{j}^{-}, s\right)\right)[f(s, z(s), z(\gamma(s)))+g(s, z(s), z(\gamma(s)))] d s \\
& \quad+\sum_{k=i(\tau)+1}^{i(\tau)+p}\left(G\left(t_{j}, t_{k}\right)-G\left(t_{j}^{-}, t_{k}\right)\right) J_{k}\left(z\left(t_{k}^{-}\right)\right) \\
& =J_{j}\left(z\left(t_{j}^{-}\right)\right) .
\end{aligned}
$$

Thus $z(t)$ is an $\omega$-periodic solution of the IDEPCAG system (2a)- $2 \mathrm{~b})$.

Conversely, suppose that $z(t)$ is an $\omega$-periodic of the IDEPCAG system (2a)-(2b). Now, we solve the IDEPCAG system 2a $-2 \mathrm{~b}$ on $I_{i(\tau)}=\left[t_{i(\tau)}, t_{i(\tau)+1}\right)$ :

$$
z^{\prime}(t)=A(t) z(t)+f\left(t, z(t), z\left(\gamma_{i(\tau)}\right)\right)+g\left(t, z(t), z\left(\gamma_{i(\tau)}\right)\right), \quad t \in\left[t_{i(\tau)}, t_{i(\tau)+1}\right)
$$

which has the solution given by

$$
z(t)=\Phi(t, \tau) z(\tau)+\int_{\tau}^{t} \Phi(t, s)\left[f\left(s, z(s), z\left(\gamma_{i(\tau)}\right)\right)+g\left(s, z(s), z\left(\gamma_{i(\tau)}\right)\right)\right] d s
$$

For $t \rightarrow t_{i(\tau)+1}$ in 21 and the impulsive effects (2b), we have

$$
\begin{aligned}
z\left(t_{i(\tau)+1}\right)=\Phi\left(t_{i(\tau)+1}, \tau\right) z(\tau)+\int_{\tau} & t_{i(\tau)+1} \Phi\left(t_{i(\tau)+1}, s\right)\left[f\left(s, z(s), z\left(\gamma_{i(\tau)}\right)\right)\right. \\
& \left.+g\left(s, z(s), z\left(\gamma_{i(\tau)}\right)\right)\right] d s+J_{i(\tau)+1}\left(z\left(t_{i(\tau)+1}^{-}\right)\right) .
\end{aligned}
$$

For $t \rightarrow t_{i(\tau)+2}$ in 21 with $\tau=t_{i(\tau)+1}$ and using 22 and the impulsive effects (2b), we obtain

$$
\begin{aligned}
z\left(t_{i(\tau)+2}\right)=\Phi\left(t_{i(\tau)+2}, \tau\right) z(\tau)+\int_{\tau}^{t_{i(\tau)+1}} & \Phi\left(t_{i(\tau)+2}, s\right)\left[f\left(s, z(s), z\left(\gamma_{i(\tau)}\right)\right)\right. \\
& \left.+g\left(s, z(s), z\left(\gamma_{i(\tau)}\right)\right)\right] d s \\
+ & \int_{t_{i(\tau)+1}}^{t_{i(\tau)+2}} \Phi\left(t_{i(\tau)+2}, s\right)\left[f\left(s, z(s), z\left(\gamma_{i(\tau)}\right)\right)+g\left(s, z(s), z\left(\gamma_{i(\tau)}\right)\right)\right] d s \\
+ & \Phi\left(t_{i(\tau)+2}, t_{i(\tau)+1}\right) J_{i(\tau)+1}\left(z\left(t_{i(\tau)+1}^{-}\right)\right)+J_{i(\tau)+2}\left(z\left(t_{i(\tau)+2}^{-}\right)\right),
\end{aligned}
$$


and in general, by induction, for any $i(t) \geq i(\tau)$ :

$$
\begin{aligned}
z(t)=\Phi(t, \tau) z(\tau)+ & \int_{\tau}^{t_{i(\tau)+1}} \Phi(t, s)\left[f\left(s, z(s), z\left(\gamma_{i(\tau)}\right)+g\left(s, z(s), z\left(\gamma_{i(\tau)}\right)\right)\right] d s\right. \\
& +\sum_{k=i(\tau)+1}^{i(t)-1} \int_{t_{k}}^{t_{k+1}} \Phi(t, s)\left[f\left(s, z(s), z\left(\gamma_{k}\right)\right)+g\left(s, z(s), z\left(\gamma_{k}\right)\right)\right] d s \\
& +\int_{t_{i(t)}}^{t} \Phi(t, s)\left[f\left(s, z(s), z\left(\gamma_{i(t)}\right)\right)+g\left(s, z(s), z\left(\gamma_{i(t)}\right)\right)\right] d s \\
& +\sum_{k=i(\tau)+1}^{i(t)} \Phi\left(t, t_{k}\right) J_{k}\left(z\left(t_{k}^{-}\right)\right) .
\end{aligned}
$$

On the other hand, one can easily see that

$$
\begin{aligned}
\int_{\tau}^{t} f(s, z(s), z(\gamma(s))) d s= & \int_{\tau}^{t_{i(\tau)+1}} f\left(s, z(s), z\left(\gamma_{i(\tau)}\right)\right) d s \\
& +\sum_{k=i(\tau)+1}^{i(t)-1} \int_{t_{k}}^{t_{k+1}} f\left(s, z(s), z\left(\gamma_{k}\right)\right) d s \\
& +\int_{t_{i(t)}}^{t} f\left(s, z(s), z\left(\gamma_{i(t)}\right)\right) d s .
\end{aligned}
$$

Then, any solution of the IDEPCAG system 2a $-2 \mathrm{~b}$ with the initial condition $z(\tau)=\xi$ can be written as

$$
\begin{aligned}
z(t)=\Phi(t, \tau) \xi & +\int_{\tau}^{t} \Phi(t, s)[f(s, z(s), z(\gamma(s)))+g(s, z(s), z(\gamma(s)))] d s \\
& +\sum_{k=i(\tau)+1}^{i(t)} \Phi\left(t, t_{k}\right) J_{k}\left(z\left(t_{k}^{-}\right)\right), \quad \tau \in \mathbb{R} .
\end{aligned}
$$

Amongst these solutions, that one will be $\omega$-periodic, for which $z(\tau)=\xi=z(\tau+\omega)$, by the condition $\left(\mathcal{N}_{\omega}\right),\left(\mathcal{P}_{2}\right)$ and using $(23)$ we obtain

$$
\begin{aligned}
\xi=(I-\Phi(\tau+\omega, \tau))^{-1}\left\{\int_{\tau}^{\tau+\omega}\right. & \Phi(\tau+\omega, s)[f(s, z(s), z(\gamma(s)))+g(s, z(s), z(\gamma(s)))] d s \\
& \left.+\sum_{k=i(\tau)+1}^{i(\tau)+p} \Phi\left(\tau+\omega, t_{k}\right) J_{k}\left(z\left(t_{k}^{-}\right)\right)\right\} .
\end{aligned}
$$


A substitution of 24 into 23 yields

$$
\begin{gathered}
z(t)=\Phi(t, \tau)(I-\Phi(\tau+\omega, \tau))^{-1} \times\left\{\int_{\tau}^{\tau+\omega} \Phi(\tau+\omega, s)[f(s, z(s), z(\gamma(s)))\right. \\
\left.+g(s, z(s), z(\gamma(s)))] d s+\sum_{k=i(\tau)+1}^{i(\tau)+p} \Phi\left(\tau+\omega, t_{k}\right) J_{k}\left(z\left(t_{k}^{-}\right)\right)\right\} \\
+\int_{\tau}^{t} \Phi(t, s)[f(s, z(s), z(\gamma(s)))+g(s, z(s), z(\gamma(s)))] d s \\
+\sum_{k=i(\tau)+1}^{i(t)} \Phi\left(t, t_{k}\right) J_{k}\left(z\left(t_{k}^{-}\right)\right) .
\end{gathered}
$$

It is easy to check the following identity using properties of the function $\Phi(t)$ :

$$
\Phi^{-1}(\tau)(I-\Phi(\tau+\omega, \tau))^{-1} \Phi(\tau+\omega)=\left(\Phi^{-1}(\tau+\omega) \Phi(\tau)-I\right)^{-1} .
$$

It follows that

$$
\begin{gathered}
z(t)=\int_{\tau}^{t} \Phi(t)\left(I+\left(\Phi^{-1}(\tau+\omega) \Phi(\tau)-I\right)^{-1}\right) \Phi(s)^{-1} \times \\
{[f(s, z(s), z(\gamma(s)))+g(s, z(s), z(\gamma(s)))] d s} \\
+\sum_{k=i(\tau)+1}^{i(\tau)+p} \Phi(t)\left(I+\left(\Phi^{-1}(\tau+\omega) \Phi(\tau)-I\right)^{-1}\right) \Phi^{-1}\left(t_{k}\right) J_{k}\left(z\left(t_{k}^{-}\right)\right) \\
+\int_{t}^{\tau+\omega} \Phi(t)\left(\Phi^{-1}(\tau+\omega) \Phi(\tau)-I\right)^{-1} \Phi^{-1}(s) \times \\
+\sum_{k=i(\tau)+1}^{i(\tau)+p}[f(s, z(s), z(\gamma(s)))+g(s, z(s), z(\gamma(s)))] d s
\end{gathered}
$$

By the Definition 3 of the Green's function for the IDEPCAG system $2 \mathrm{a}-2 \mathrm{2b}$, we give

$$
\begin{aligned}
z(t)=\int_{\tau}^{\tau+\omega} & G(t, s)[f(s, z(s), z(\gamma(s)))+g(s, z(s), z(\gamma(s)))] d s \\
& +\sum_{k=i(\tau)+1}^{i(\tau)+p} G\left(t, t_{k}\right) J_{k}\left(z\left(t_{k}^{-}\right)\right) .
\end{aligned}
$$


Hence, the $\omega$-periodic solution of the IDEPCAG system (2a)-2b) satisfies the integral equation system (16). The proof is complete.

In such a case the IDEPCAG system (2a)-(2b) has an $\omega$-periodic solution $z(t)$ given by the integral equation system (16). Before studying the existence of solutions of integral equation system (16), firstly, we define the Green's operator for impulsive periodic solutions of the IDEPCAG system (2a)-(2b).

Consider the Green's operator $\Im: \mathcal{P C}_{\omega} \rightarrow \mathcal{P C} \mathcal{C}_{\omega}$ by

$$
\begin{aligned}
(\Im z)(t)= & \int_{\tau}^{\tau+\omega} G(t, s)[f(s, z(s), z(\gamma(s)))+g(s, z(s), z(\gamma(s)))] d s \\
& +\sum_{k=i(\tau)+1}^{i(\tau)+p} G\left(t, t_{k}\right) J_{k}\left(z\left(t_{k}^{-}\right)\right) .
\end{aligned}
$$

It is easy to see that the IDEPCAG system (2a)-(2b) has an $\omega$-periodic solution if and only if the Green's operator $\Im$ has one fixed point in $\mathcal{P C} \omega$.

To prove some existence criteria for $\omega$-periodic solutions of the IDEPCAG system 2a)-2b we use the Banach, Schauder and Krasnoselskii's fixed point theorem.

Next we state first Krasnoselskii's fixed point theorem which enables us to prove the existence of a periodic solution. For the proof of Krasnoselskii's fixed point theorem we refer the reader to [34].

Theorem A (Krasnoselskii's fixed point theorem):

Let $S$ be a closed convex nonempty subset of a Banach space $(E,\|\cdot\|)$. Suppose that $\mathcal{A}$ and $\mathcal{B}$ map $S$ into $E$ such that

(i) $x, y \in S$, implies $\mathcal{A} x+\mathcal{B} y \in S$,

(ii) $\mathcal{A}$ is a contraction mapping,

(iii) $\mathcal{B}$ is completely continuous.

Then there exists $z \in S$ with $z=\mathcal{A} z+\mathcal{B} z$.

Remark 8. Krasnoselskii's theorem may be combined with Banach and Schauder's fixed point theorems. In a certain sense, we can interpret this as follows: if a compact operator has the fixed point property, under a small perturbation, then this property can be inherited. The theorem is useful in establishing the existence results for perturbed operator equations. It also has a wide range of applications to nonlinear integral equations of mixed type for proving the existence of solutions. Thus the existence of fixed points for the sum of two operators has attracted tremendous interest, and their applications are frequent in nonlinear analysis. See [4, 32, 34].

We note that to apply Krasnoselskii's fixed point theorem we need to construct two mappings; one is a contraction mapping and the other is completely continuous. Therefore, we express the Green's operator (26) as

$$
(\Im z)(t)=(\mathcal{A} z)(t)+(\mathcal{B} z)(t),
$$


where $\mathcal{A}, \mathcal{B}: \mathcal{P C} \mathcal{C}_{\omega} \rightarrow \mathcal{P C} \mathcal{C}_{\omega}$ are given by

$$
(\mathcal{A} z)(t)=\int_{\tau}^{\tau+\omega} G(t, s) f(s, z(s), z(\gamma(s))) d s
$$

and

$$
(\mathcal{B} z)(t)=\int_{\tau}^{\tau+\omega} G(t, s) g(s, z(s), z(\gamma(s))) d s+\sum_{k=i(\tau)+1}^{i(\tau)+p} G\left(t, t_{k}\right) J_{k}\left(z\left(t_{k}^{-}\right)\right) .
$$

To simplify notations, we introduce the following constant and sets.

$c_{G}=\max _{t, s \in[\tau, \tau+\omega]}|G(t, s)|, \quad \mathbb{S}=\left\{z \in \mathcal{P} \mathcal{C}_{\omega}:\|z\| \leq \mathcal{R}\right\} \quad$ and $\quad \mathcal{C}_{\mathcal{R}}=\left\{z \in \mathbb{R}^{n}:\|z\| \leq \mathcal{R}\right\}$.

Lemma 9. If $\left(\mathcal{N}_{\omega}\right),(\mathcal{P})$ and $\left(\mathcal{L}_{f}\right)$ hold, $\mathcal{A}$ is given by 27] with $c_{G} L_{1}<1$, then $\mathcal{A}$ is a contraction mapping.

Proof. Let $\mathcal{A}$ be defined by $(27)$. First we want to show that $(\mathcal{A} \varphi)(t+\omega)=(\mathcal{A} \varphi)(t)$. Let $\varphi \in \mathcal{P C}_{\omega}$. Then using (27), the periodicity of Green's function and Remark 6 (ii), we obtain

$$
\begin{aligned}
(\mathcal{A} \varphi)(t+\omega) & =\int_{\tau+\omega}^{\tau+2 \omega} G(t+\omega, s) f(s, z(s), z(\gamma(s))) d s \\
& =\int_{\tau}^{\tau+\omega} G(t+\omega, s+\omega) f(s+\omega, z(s+\omega), z(\gamma(s+\omega))) d s \\
& =\int_{\tau}^{\tau+\omega} G(t+\omega, s+\omega) f(s+\omega, z(s+\omega), z(\gamma(s)+\omega)) d s \\
& =\int_{\tau}^{\tau+\omega} G(t, s) f(s, z(s), z(\gamma(s))) d s=(\mathcal{A} \varphi)(t) .
\end{aligned}
$$

Secondly, we show that is $\mathcal{A}$ is a contraction mapping.

Let any $\varphi, \zeta \in \mathcal{P C}_{\omega}$. By using $\left(\mathcal{L}_{f}\right)$ then for any $t \in[\tau, \tau+\omega]$, we get

$$
\begin{aligned}
\|\mathcal{A} \varphi-\mathcal{A} \zeta\| & =\sup _{t \in[\tau, \tau+\omega]}|\mathcal{A} \varphi(t)-\mathcal{A} \zeta(t)| \\
& \leq \sup _{t \in[\tau, \tau+\omega]} \int_{\tau}^{\tau+\omega}|G(t, s)||f(s, \varphi(s), \varphi(\gamma(s)))-f(s, \zeta(s), \zeta(\gamma(s)))| d s \\
& \leq \int_{\tau}^{\tau+\omega} c_{G}\left[p_{1}(s)|\varphi(s)-\zeta(s)|+p_{2}(s)|\varphi(\gamma(s))-\zeta(\gamma(s))|\right] d s \\
& \leq c_{G}\left(\int_{\tau}^{\tau+\omega}\left[p_{1}(s)+p_{2}(s)\right] d s\right)\|\varphi-\zeta\| \\
& \leq c_{G} L_{1}\|\varphi-\zeta\| .
\end{aligned}
$$

Hence $\mathcal{A}$ defines a contraction mapping. 
Using the same method, one can show that $\mathcal{B}$ is given by $(28$, may be also a contraction operator.

Lemma 10. If $\left(\mathcal{N}_{\omega}\right),(\mathcal{P}),\left(\mathcal{L}_{g}\right)$ and $\left(\mathcal{L}_{J}\right)$ hold, $\mathcal{B}$ is given by 28 with $c_{G}\left(L_{2}+\right.$ $\left.L_{3}\right)<1$, then $\mathcal{B}$ is a contraction mapping.

Lemma 11. If $\mathcal{B}$ is defined by 28 , then $\mathcal{B}$ is completely continuous, i.e., $\mathcal{B}$ is continuous and the image of $\mathcal{B}$ is contained in a compact set.

Proof. The proof of the operator $\mathcal{B}$ is completely continuous is given in the following steps.

Step 1: First we prove that $\mathcal{B}: \mathcal{P C} \mathcal{C}_{\omega} \rightarrow \mathcal{P C} \mathcal{C}_{\omega}$ is continuous.

As the operator $\mathcal{A}$, a change of variable in 28 we have $(\mathcal{B} \varphi)(t+\omega)=(\mathcal{B} \varphi)(t)$. Now, we want to show $\mathcal{B}$ is continuous.

The function $g(t, x, y)$ is uniformly continuous on $[\tau, \tau+\omega] \times \mathcal{C}_{\mathcal{R}} \times \mathcal{C}_{\mathcal{R}}$ and by the periodicity in $t$, the function $g(t, x, y)$ is uniformly continuous on $\mathbb{R} \times$ $\mathcal{C}_{\mathcal{R}} \times \mathcal{C}_{\mathcal{R}}$. The functions $J_{k}$ are uniformly continuous on $\mathcal{C}_{\mathcal{R}}$. Thus, for any $\epsilon^{\prime}=\frac{\epsilon}{c_{G}(\omega+p)}>0$, there exists $\delta=\delta(\epsilon)>0$ such that $z_{1}, z_{2} \in \mathbb{S},\left\|z_{1}-z_{2}\right\| \leq$ $\delta$ implies $\left|g\left(t, z_{1}(t), z_{1}(\gamma(t))\right)-g\left(t, z_{2}(t), z_{2}(\gamma(t))\right)\right| \leq \epsilon^{\prime}$ for $t \in[\tau, \tau+\omega]$ and $\left|J_{k}\left(z_{1}\right)-J_{k}\left(z_{2}\right)\right| \leq \epsilon^{\prime}$. Then $\left\|\mathcal{B} z_{1}-\mathcal{B} z_{2}\right\| \leq \epsilon$.

In fact, by the continuity of $g$ and $J_{k}$, for $t \in[\tau, \tau+\omega]$ and $z_{1}, z_{2} \in \mathbb{S}$, we have

$$
\begin{gathered}
\left|g\left(t, z_{1}(t), z_{1}(\gamma(t))\right)-g\left(t, z_{2}(t), z_{2}(\gamma(t))\right)\right| \leq \epsilon^{\prime} \\
\left|J_{k}\left(z_{1}\right)-J_{k}\left(z_{2}\right)\right| \leq \epsilon^{\prime},
\end{gathered}
$$

and then

$$
\begin{aligned}
\left\|\mathcal{B} z_{1}-\mathcal{B} z_{2}\right\|= & \sup _{t \in[\tau, \tau+\omega]}\left|\mathcal{B} z_{1}(t)-\mathcal{B} z_{2}(t)\right| \\
\leq & \sup _{t \in[\tau, \tau+\omega]} \int_{\tau}^{\tau+\omega}|G(t, s)|\left|g\left(s, z_{1}(s), z_{1}(\gamma(s))\right)-g\left(s, z_{2}(s), z_{2}(\gamma(s))\right)\right| d s \\
& \quad+\sup _{t \in[\tau, \tau+\omega]} \sum_{k=i(\tau)+1}^{i(\tau)+p}\left|G\left(t, t_{k}\right)\right|\left|J_{k}\left(z_{1}\left(t_{k}^{-}\right)\right)-J_{k}\left(z_{2}\left(t_{k}^{-}\right)\right)\right| \\
& \leq \int_{\tau}^{\tau+\omega} c_{G} \epsilon^{\prime} d s+c_{G} \cdot p \epsilon^{\prime} \leq c_{G}(\omega+p) \epsilon^{\prime}=\epsilon
\end{aligned}
$$

Thus the operator $\mathcal{B}$ is continuous.

Step 2: To show that the image of $\mathcal{B}$ is contained in a compact set.

Let $x, y \in \mathcal{C}_{\mathcal{R}}$ and $s \in[\tau, \tau+\omega]$, for the continuity of the function $g(s, x, y)$ and $J_{k}(x), k \in\left\{i_{(\tau)+1}, \ldots, i_{(\tau)+p}\right\}$, there exists $M_{i}>0, i=1,2$, such that $|g(s, x, y)| \leq$ 
$M_{1}$ and $\left|J_{k}(x)\right| \leq M_{2}$. Let $\varphi_{n} \in \mathbb{S}$ where $n$ is a positive integer, then we have

$$
\begin{aligned}
\left\|\mathcal{B} \varphi_{n}\right\|= & \sup _{t \in[\tau, \tau+\omega]}\left|\mathcal{B} \varphi_{n}(t)\right| \leq \sup _{t \in[\tau, \tau+\omega]} \int_{\tau}^{\tau+\omega}|G(t, s)|\left|g\left(s, \varphi_{n}(s), \varphi_{n}(\gamma(s))\right)\right| d s \\
& +\sup _{t \in[\tau, \tau+\omega]} \sum_{k=i(\tau)+1}^{i(\tau)+p}\left|G\left(t, t_{k}\right)\right|\left|J_{k}\left(\varphi_{n}\left(t_{k}^{-}\right)\right)\right| \\
\leq & c_{G}\left\{\int_{\tau}^{\tau+\omega}\left|g\left(s, \varphi_{n}(s), \varphi_{n}(\gamma(s))\right)\right| d s+\sum_{k=i(\tau)+1}^{i(\tau)+p}\left|J_{k}\left(\varphi_{n}\left(t_{k}^{-}\right)\right)\right|\right\} \\
\leq & c_{G}\left(\omega M_{1}+p M_{2}\right) .
\end{aligned}
$$

Moreover, a direct calculation $\left(\mathcal{B} \varphi_{n}(t)\right)^{\prime}$ shows that

$$
\begin{aligned}
\left(\mathcal{B} \varphi_{n}(t)\right)^{\prime}= & \left(\int_{\tau}^{\tau+\omega} G(t, s) g\left(s, \varphi_{n}(s), \varphi_{n}(\gamma(s))\right) d s+\sum_{k=i(\tau)+1}^{i(\tau)+p} G\left(t, t_{k}\right) J_{k}\left(\varphi_{n}\left(t_{k}^{-}\right)\right)\right)^{\prime} \\
= & A(t)\left(\int_{\tau}^{\tau+\omega} G(t, s) g\left(s, \varphi_{n}(s), \varphi_{n}(\gamma(s))\right) d s\right)+g\left(t, \varphi_{n}(t), \varphi_{n}(\gamma(t))\right) \\
& \quad+A(t) \sum_{k=i(\tau)+1}^{i(\tau)+p} G\left(t, t_{k}\right) J_{k}\left(\varphi_{n}\left(t_{k}^{-}\right)\right) \\
= & A(t) \mathcal{B} \varphi_{n}(t)+g\left(t, \varphi_{n}(t), \varphi_{n}(\gamma(t))\right) .
\end{aligned}
$$

As $A(t)$ is bounded on $[\tau, \tau+\omega]$ and $\mathcal{B} \varphi_{n}(t), g\left(t, \varphi_{n}(t), \varphi_{n}(\gamma(t))\right.$ are bounded on $\mathbb{S}$ and $[\tau, \tau+\omega] \times \mathbb{S} \times \mathbb{S}$, respectively. Thus, the above expression yields $\left\|\left(\mathcal{B} \varphi_{n}\right)^{\prime}\right\| \leq L$, for some positive constant $L$. Hence the sequence $\left(\mathcal{B} \varphi_{n}\right)$ is uniformly bounded and equi-continuous. The Ascoli-Arzela's theorem implies that a subsequence $\left(\mathcal{B} \varphi_{n_{k}}\right)$ of $\left(\mathcal{B} \varphi_{n}\right)$ converges uniformly to a continuous $\omega$-periodic function. Thus $\mathcal{B}$ is continuous and $\mathcal{B}(\mathbb{S})$ is a compact set.

In a similar way, for $\mathcal{A}$ we obtain the following lemma.

Lemma 12. If $\mathcal{A}$ is defined by (27), then $\mathcal{A}$ is completely continuous.

Theorem 13. Assume that the hypotheses $\left(\mathcal{N}_{\omega}\right),(\mathcal{P}),\left(\mathcal{L}_{f}\right),\left(\mathcal{M}_{g}\right)$ and $\left(\mathcal{M}_{J}\right)$ are satisfied and let $\mathcal{R}$ be a positive constant satisfying the inequality

$$
c_{G}\left(L_{1}+C_{2}+C_{3}\right) \mathcal{R}+c_{G}\left(\left(\alpha+\rho_{2}\right) \omega+\rho_{3}\right) \leq \mathcal{R} .
$$

Then the IDEPCAG system (2a)-2b) has at least one $\omega$-periodic solution in $\mathbb{S}$.

Proof. By Lemma 9, the mapping $\mathcal{A}$ is a contraction and it is clear that $\mathcal{A}: \mathcal{P C}_{\omega} \rightarrow$ $\mathcal{P C}{ }_{\omega}$.

Also, from Lemma $11, \mathcal{B}$ is completely continuous.

Next, we prove that if $\varphi, \zeta \in \mathbb{S}$ with $\|\varphi\| \leq \mathcal{R}$ and $\|\zeta\| \leq \mathcal{R}$, then $\|\mathcal{A} \phi+\mathcal{B} \zeta\| \leq \mathcal{R}$. 
Let $\varphi, \zeta \in \mathbb{S}$ with $\|\varphi\| \leq \mathcal{R}$ and $\|\zeta\| \leq \mathcal{R}$.

Then

$$
\begin{gathered}
\|\mathcal{A} \varphi+\mathcal{B} \zeta\| \leq \sup _{t \in[\tau, \tau+\omega]} \int_{\tau}^{\tau+\omega}|G(t, s)| \mid f(s, \varphi(s), \varphi(\gamma(s))-f(s, 0,0) \mid d s \\
+\sup _{t \in[\tau, \tau+\omega]} \int_{\tau}^{\tau+\omega}|G(t, s)||f(s, 0,0)| d s \\
\quad+\sup _{t \in[\tau, \tau+\omega]} \int_{\tau}^{\tau+\omega}|G(t, s)||g(s, \zeta(s), \zeta(\gamma(s)))| d s \\
\quad+\sup _{t \in[\tau, \tau+\omega]} \sum_{k=i(\tau)+1}^{i(\tau)+p}\left|G\left(t, t_{k}\right)\right|\left|J_{k}\left(\zeta\left(t_{k}^{-}\right)\right)\right| \\
\left.\leq c_{G}\left(\int_{\tau}^{\tau+\omega} p_{1}(s)+p_{1}(s)\right] d s\right)\|\varphi\|+\alpha c_{G} \omega \\
+c_{G}\left(\int_{\tau}^{\tau+\omega}\left[\kappa_{1}(s)+\kappa_{1}(s)\right] d s\right)\|\zeta\|+\rho_{2} c_{G} \omega \\
+c_{G}\left(\sum_{k=i(\tau)+1}^{i(\tau)+p} \varrho_{k}(t)\right)\|\zeta\|+\rho_{3} c_{G} \\
\leq c_{G}\left(L_{1}+C_{2}+C_{3}\right) \mathcal{R}+c_{G}\left(\left(\alpha+\rho_{2}\right) \omega+\rho_{3}\right) .
\end{gathered}
$$

We now see that all the conditions of Krasnoselskii's theorem are satisfied. Thus there exists a fixed point $z$ in $\mathbb{S}$ such that $z=\mathcal{A} z+\mathcal{B} z$. By Proposition 7 , this fixed point is a solution of the IDEPCAG system 2a $-2 \mathrm{~b}$. Hence the IDEPCAG system 2a $-2 \mathrm{~b}$ has a $\omega$-periodic solution. The proof is completed.

By the symmetry of the conditions, we will obtain as Theorem 13 .

Theorem 14. Assume that the hypotheses $\left(\mathcal{N}_{\omega}\right),(\mathcal{P}),\left(\mathcal{M}_{f}\right)\left(\mathcal{L}_{g}\right)$ and $\left(\mathcal{L}_{J}\right)$ are satisfied and let $\mathcal{R}$ be a positive constant satisfying the inequality

$$
c_{G}\left(C_{1}+L_{2}+L_{3}\right) \mathcal{R}+c_{G}\left(\left(\beta+\rho_{1}\right) \omega+\kappa\right) \leq \mathcal{R} .
$$

Then the IDEPCAG system (2a)-22b) has at least one $\omega$-periodic solution in $\mathbb{S}$.

Applying the Banach's fixed point theorem we have:

Theorem 15. Assume that the hypotheses $\left(\mathcal{N}_{\omega}\right),(\mathcal{P}),\left(\mathcal{L}_{f}\right),\left(\mathcal{L}_{g}\right)$ and $\left(\mathcal{L}_{J}\right)$ are satisfied and let

$$
c_{G}\left(L_{1}+L_{2}+L_{3}\right)<1 .
$$

Then the IDEPCAG system 2a)-2b has a unique $\omega$-periodic solution.

Proof. Let the mapping $\Im$ be given by 26]. For $\varphi, \zeta \in \mathcal{P C} \mathcal{C}_{\omega}$, in view of 26 , we have 


$$
\begin{aligned}
&\|\Im \varphi-\Im \zeta\| \leq \sup _{t \in[\tau, \tau+\omega]} \int_{\tau}^{\tau+\omega}|G(t, s)||f(s, \varphi(s), \varphi(\gamma(s)))-f(s, \zeta(s), \zeta(\gamma(s)))| d s \\
&+\sup _{t \in[\tau, \tau+\omega]} \int_{\tau}^{\tau+\omega}|G(t, s)||g(s, \varphi(s), \varphi(\gamma(s)))-g(s, \zeta(s), \zeta(\gamma(s)))| d s \\
& \quad+\sup _{t \in[\tau, \tau+\omega]} \sum_{k=i(\tau)+1}^{i(\tau)+p}\left|G\left(t, t_{k}\right)\right|\left|J_{k}\left(\varphi\left(t_{k}^{-}\right)\right)-J_{k}\left(\zeta\left(t_{k}^{-}\right)\right)\right| \\
& \leq c_{G}\left(L_{1}+L_{2}+L_{3}\right)\|\varphi-\zeta\| .
\end{aligned}
$$

This completes the proof by invoking the contraction mapping principle.

As a direct consequence of the method, Schauder's theorem imply:

Theorem 16. Suppose the hypotheses $\left(\mathcal{N}_{\omega}\right),(\mathcal{P}),\left(\mathcal{M}_{f}\right),\left(\mathcal{M}_{g}\right)$ and $\left(\mathcal{M}_{J}\right)$ hold. Let $R$ be a positive constant satisfying the inequality

$$
c_{G}\left(C_{1}+C_{2}+C_{3}\right) \mathcal{R}+c_{G}\left(\left(\rho_{1}+\rho_{2}\right) \omega+\rho_{3}\right) \leq \mathcal{R} .
$$

Then the IDEPCAG system (2a)-2b) has at least one $\omega$-periodic solution in $\mathbb{S}$.

To determine criteria for the existence and uniqueness of subharmonic solutions of the IDEPCAG system 2a - 2b], from now on we make the assumption:

$\left(\mathcal{P}_{\omega}\right)$ There exists $\omega=$ l.c.m. $\left\{\omega_{1}, \omega_{2}, \omega_{3}, \omega_{4}\right\}>0, \frac{\omega_{i}}{\omega_{j}}$ is a rational number for all $i, j=1,2,3,4$ such that:

(1) $A(t), f\left(t, x_{1}, y_{1}\right)$ and $g\left(t, x_{2}, y_{2}\right)$ are periodic functions in $t$ with a period $\omega_{1}, \omega_{2}$ and $\omega_{3}$, respectively, for all $t \geq \tau$.

(2) There exists $p \in \mathbb{Z}^{+}$, for which the sequences $\left\{t_{i}\right\}_{i \in \mathbb{Z}},\left\{\gamma_{i}\right\}_{i \in \mathbb{Z}}$, satisfy the $\left(\omega_{4}, p\right)$ condition.

(3) The sequence $\left\{J_{i}\right\}_{i \in \mathbb{Z}}$ satisfies $J_{i+p}=J_{i}$, some positive integer $p \in \mathbb{N}$.

As immediate corollaries of Theorems 13, 16, the following results are true.

Corollary 17. Suppose the hypotheses $\left(\mathcal{N}_{\omega}\right),\left(\mathcal{P}_{\omega}\right),\left(\mathcal{L}_{f}\right),\left(\mathcal{M}_{g}\right),\left(\mathcal{M}_{J}\right)$ and 30 hold. Then the IDEPCAG system (2a)-2b) has at least one subharmonic solution in $\mathbb{S}$.

Corollary 18. Suppose the hypotheses $\left(\mathcal{N}_{\omega}\right),\left(\mathcal{P}_{\omega}\right),\left(\mathcal{M}_{f}\right),\left(\mathcal{L}_{g}\right),\left(\mathcal{L}_{J}\right)$ and 31 ) hold. Then the IDEPCAG system (2a)-2b) has at least one subharmonic solution in $\mathbb{S}$.

Corollary 19. Suppose the hypotheses $\left(\mathcal{N}_{\omega}\right),\left(\mathcal{P}_{\omega}\right),\left(\mathcal{L}_{f}\right),\left(\mathcal{L}_{g}\right),\left(\mathcal{L}_{J}\right)$ and 32 hold. Then the IDEPCAG system (2a)-(2b) has a unique subharmonic solution.

Corollary 20. Suppose the hypotheses $\left(\mathcal{N}_{\omega}\right),\left(\mathcal{P}_{\omega}\right),\left(\mathcal{M}_{f}\right),\left(\mathcal{M}_{g}\right),\left(\mathcal{M}_{J}\right)$ and 33 ) hold. Then the IDEPCAG system 2a - 2b has at least one subharmonic solution in $\mathbb{S}$. 


\section{Applications and Illustrative examples}

We will introduce appropriate examples in this section. These examples will show the feasibility of our theory.

Mathematical modelling of real-life problems usually results in functional equations, like ordinary or partial differential equations, integral and integro-differential equations, stochastic equations. Many mathematical formulation of physical phenomena contain integro-differential equations, these equations arises in many fields like fluid dynamics, biological models and chemical kinetics. So, we first consider nonlinear impulsive integro-differential equations with piecewise alternately advanced and retarded argument mentioned in the introduction and obtain some new sufficient conditions for the existence of the impulsive periodic solutions of these systems.

Example 21. Consider the following nonlinear impulsive integro-differential equations with piecewise alternately advanced and retarded argument of generalized type:

$$
\begin{aligned}
& z^{\prime}(t)=A(t) z(t)+\int_{-\infty}^{t} C(t, s, z(\gamma(s))) d s+g(t, z(t), z(\gamma(t))), \quad t \neq t_{k}, \\
& \left.\Delta z\right|_{t_{k}}=\mathcal{J}_{k}\left(z\left(t_{k}^{-}\right)\right), \quad k \in \mathbb{Z},
\end{aligned}
$$

where, the sequences $\left\{t_{i}\right\}_{i \in \mathbb{Z}}$ and $\left\{\gamma_{i}\right\}_{i \in \mathbb{Z}}$, satisfy the $(\omega, p)$ condition, $\mathcal{J}_{k+p}=\mathcal{J}_{k}$, $A(t)$ is a continuous matrix on $\mathbb{R}, g: \mathbb{R} \times \mathbb{R}^{n} \times \mathbb{R}^{n} \rightarrow \mathbb{R}^{n}$ and $C: \mathbb{R} \times \mathbb{R} \times \mathbb{R}^{n} \rightarrow \mathbb{R}^{n}$ are $\omega$-periodic continuous functions, i.e., for $x, y \in \mathbb{R}^{n}$ fixed, $g(t+\omega, x, y)=g(t, x, y)$ and $C(t+\omega, s+\omega, y)=C(t, s, y)$. Moreover, we will refer to the following specific conditions.

$\left(\mathcal{L}_{C}\right)$ There exists a continuous function $\lambda: \mathbb{R}^{2} \rightarrow[0, \infty)$ such that: $t, s \in \mathbb{R}$ and for any $y_{1}, y_{2} \in \mathbb{R}^{n}$ we have

$$
\left|C\left(t, s, y_{1}\right)-C\left(t, s, y_{2}\right)\right| \leq \lambda(t, s)\left|y_{1}-y_{2}\right|
$$

and $\sup _{t \in \mathbb{R}} \int_{-\infty}^{t} \lambda(t, s) d s=M, M \omega<L_{4}, \sup _{t \in \mathbb{R}} \int_{-\infty}^{t} \lambda(t, s, 0) d s=\mu$.

$\left(\mathcal{M}_{C}\right)$ For every $\mathcal{R}>0, t, s \in \mathbb{R},|y| \leq \mathcal{R}$, there exist $\lambda, \beta: \mathbb{R}^{2} \rightarrow[0, \infty)$ functions and positive constants $\lambda_{3}$, $\varsigma$ with $\lambda_{3} \omega \leq C_{4}$ for which

$$
|C(t, s, y)| \leq \lambda(t, s)|y|+\beta(t, s),
$$

where $\sup _{t \in \mathbb{R}} \int_{-\infty}^{t} \lambda(t, s) d s=\lambda_{3}$, $\sup _{t \in \mathbb{R}} \int_{-\infty}^{t} \beta(t, s) d s=\varsigma$.

(C) Let $\mathcal{R}>0, t, s \in \mathbb{R}$ and $y_{1}, y_{2} \in \mathbb{R}^{n},\left|y_{i}\right| \leq \mathcal{R}, i=1,2$. For any $\epsilon>0$ there exist $\delta>0$ and $\lambda: \mathbb{R}^{2} \rightarrow[0, \infty)$ a function such that $\left|y_{1}-y_{2}\right| \leq \delta$ implies

$$
\left|C\left(t, s, y_{1}\right)-C\left(t, s, y_{2}\right)\right| \leq \epsilon \lambda(t, s), \quad t, s \in \mathbb{R},
$$

where $\sup _{t \in \mathbb{R}} \int_{-\infty}^{t} \lambda(t, s) d s=\lambda_{3}<\infty$. 
Assume that the hypotheses $\left(\mathcal{N}_{\omega}\right),(\mathcal{C}),\left(\mathcal{L}_{C}\right),\left(\mathcal{M}_{g}\right)$ and $\left(\mathcal{M}_{J}\right)$ are satisfied and

$$
c_{G}\left(L_{4}+C_{2}+C_{3}\right) \mathcal{R}+c_{G}\left(\left(\mu+\rho_{2}\right) \omega+\rho_{3}\right) \leq \mathcal{R} .
$$

Hence, by Theorem 13, the nonlinear impulsive integro-differential with the IDEPCAG system (34a)-(34b) has at least one $\omega$-periodic solution.

Suppose the the hypotheses $\left(\mathcal{N}_{\omega}\right),(\mathcal{C}),\left(\mathcal{M}_{C}\right),\left(\mathcal{L}_{g}\right)$, and $\left(\mathcal{L}_{J}\right)$ are satisfied and

$$
c_{G}\left(C_{4}+L_{2}+L_{3}\right) \mathcal{R}+c_{G}((\beta+\varsigma) \omega+\kappa) \leq \mathcal{R} .
$$

Then, by Theorem 14, the nonlinear impulsive integro-differential with the IDEPCAG system (34a)-(34b) has at least one w-periodic solution.

If the hypotheses $\left(\overline{\mathcal{N}}_{\omega}\right),(\mathcal{C}),\left(\mathcal{L}_{C}\right),\left(\mathcal{L}_{g}\right)$ and $\left(\mathcal{L}_{J}\right)$ are satisfied and let

$$
c_{G}\left(L_{4}+L_{2}+L_{3}\right)<1 .
$$

Then the nonlinear impulsive integro-differential with the IDEPCAG system (34a)(34b) has a unique $\omega$-periodic solution.

Second example, we consider impulsive hybrid neural networks are widely investigated in the field of nonlinear dynamics, and used to model many applications in different areas such as pattern recognition, associative memory, and combinatorial optimization. We construct the example for a model of impulsive neural network with piecewise alternately advanced and retarded generalized argument below.

Example 22. Consider the following impulsive cellular neural networks with piecewise alternately advanced and retarded argument of generalized type:

$$
\begin{aligned}
& z_{i}^{\prime}(t)=-a_{i}(t) z_{i}(t)+\sum_{j=1}^{m}\left[b_{i j}(t) f_{j}\left(z_{j}(t)\right)+c_{i j}(t) g_{j}\left(z_{j}(\gamma(t))\right)\right]+d_{i}(t), t \neq t_{k}, \\
& \left.\Delta z\right|_{t_{k}}=\mathcal{J}_{k}\left(z\left(t_{k}^{-}\right)\right), \quad k \in \mathbb{Z},
\end{aligned}
$$

where $a_{i}, b_{i j}, c_{i j}$ and $d_{i}, 1 \leq i \leq m$ are positive $\omega_{1}$-periodic functions, $f_{j}$ is $\omega_{2}$ periodic function and $g_{j}$ is $\omega_{3}$-periodic function, $\gamma(t)=\gamma_{k}$, if $t_{k} \leq t<t_{k+1}, k \in \mathbb{Z}$, the sequences $\left\{t_{k}\right\}_{k \in \mathbb{Z}}$ and $\left\{\gamma_{k}\right\}_{k \in \mathbb{Z}}$ satisfy the $\left(\omega_{4}, p\right)$ condition and $\mathcal{J}_{k+p}=\mathcal{J}_{k}$, $p \in \mathbb{N}, k \in \mathbb{Z}$.

Moreover, we shall introduce the following assumptions:

$\left(\mathcal{L G}_{f}\right) \quad f_{j}: \mathbb{R} \rightarrow \mathbb{R}$ is a continuous function for $1 \leq j \leq m$ such that

$$
\left|f_{j}(x)-f_{j}(y)\right| \leq \mathcal{L}_{f}|x-y|
$$

where $\mathcal{L}_{f} \in \mathbb{R}_{+}$and $f_{j}(t, 0)=0$.

$\left(\mathcal{L G}_{g}\right) g_{j}: \mathbb{R} \rightarrow \mathbb{R}$ is a continuous function for $1 \leq j \leq m$ such that

$$
\left|g_{j}(x)-g_{j}(y)\right| \leq \mathcal{L}_{g}|x-y|,
$$

where $\mathcal{L}_{g} \in \mathbb{R}_{+}$and $g_{j}(t, 0)=0$.

$\left(\mathcal{H}_{g}\right)$ For every $\mathcal{R}>0, t \in \mathbb{R},|x| \leq \mathcal{R}$, there exists positive function $\ell$ for which

$$
\left|g_{j}(t, x)\right| \leq \ell(t)|x|, 1 \leq j \leq m,
$$


where $\max _{1 \leq i \leq m} \sum_{j=1}^{m}\left(\int_{\tau}^{\tau+\omega}\left[c_{i j}(s) \ell(s)\right] d s\right) \leq \mathcal{C}_{1}, \omega \in \mathbb{R}_{+}, \tau \in \mathbb{R}$.

$\left(\mathcal{H}_{J}\right)$ For every $\mathcal{R}>0, t \in \mathbb{R},|x| \leq \mathcal{R}$, there exist functions $\varrho_{k}: \mathbb{R} \rightarrow[0, \infty)$, $k \in \mathbb{Z}$ and positive constant $\mathcal{C}_{2}$ for which

$$
\left|J_{k}(x)\right| \leq \varrho_{k}(t)|x|
$$

where $\sum_{k=i(\tau)+1}^{i(\tau)+p} \varrho_{k}(t) \leq \mathcal{C}_{2}, p \in \mathbb{R}_{+}, \tau \in \mathbb{R}$

Let us define

$$
\begin{gathered}
\omega=\text { l.c.m. }\left\{\omega_{1}, \omega_{2}, \omega_{3}, \omega_{4}\right\}, \quad \bar{b}:=\max _{1 \leq i \leq m} \sum_{j=1}^{m}\left(\sup _{t \in[\tau, \tau+\omega]} b_{i j}(t)\right) \omega, \\
\bar{c}:=\max _{1 \leq i \leq m} \sum_{j=1}^{m}\left(\sup _{t \in[\tau, \tau+\omega]} c_{i j}(t)\right) \omega \quad \text { and } \quad \bar{d}:=\max _{1 \leq i \leq m}\left(\sup _{t \in[\tau, \tau+\omega]} d_{i}(t)\right) .
\end{gathered}
$$

As $a_{i}$ is a positive periodic function, it is easy to see the condition $\left(\mathcal{N}_{\omega}\right)$ is satisfied. If the hypotheses $\left(\mathcal{L G}_{f}\right),\left(\mathcal{H}_{g}\right)$ and $\left(\mathcal{H}_{J}\right)$ are satisfied and

$$
c_{G}\left(\bar{b} \mathcal{L}_{f}+\mathcal{C}_{1}+\mathcal{C}_{2}\right) \mathcal{R}+c_{G} \bar{d} \omega \leq \mathcal{R} .
$$

Hence, by Corollary 17, the IDEPCAG system (35a)-(35b) has at least one $\omega$ periodic solution, i.e., a subharmonic solution.

If $\left(\mathcal{L G}_{f}\right),\left(\mathcal{L G}_{g}\right)$ and $\left(\mathcal{L}_{J}\right)$ are satisfied and

$$
c_{G}\left(\bar{b} L_{f}+\bar{c} L_{g}+L_{3}\right)<1 .
$$

Then, by Corollary 19, the IDEPCAG system (35a)-35b has a unique subharmonic solution.

Let us consider another example for impulsive second-order differential equations with piecewise alternately advanced and retarded argument. In this case, we can show the existence and uniqueness of periodic solutions following the result of this paper.

Example 23. Consider the following impulsive second-order differential equations with piecewise alternately advanced and retarded argument:

$y^{\prime \prime}(t)+\left(\kappa_{2} y^{2}(t)+3\right) y^{\prime}(t)+4 y(t)-\kappa_{1} \sin (\pi t) y^{2}\left(2\left[\frac{t+1}{2}\right]\right)-\kappa_{2} \cos (\pi t)=0, t \neq t_{k}$

$\left.\Delta y\right|_{t_{k}}=a(t) \cdot y\left(t_{k}^{-}\right),\left.\quad \Delta y^{\prime}\right|_{t_{k}}=b(t) \cdot y^{\prime}\left(t_{k}^{-}\right), \quad k \in \mathbb{Z}$,

where $\kappa_{1}, \kappa_{2} \in \mathbb{R}, a(\cdot)$ and $b(\cdot)$ are 2 -periodic functions.

According to Eq.37a), we have $\gamma(t)=2\left[\frac{t+1}{2}\right]$, then $t_{j}=2 j-1, \gamma_{j}=2 j$ for all $j \in \mathbb{Z}$. Therefore the sequences $\left\{t_{i}\right\}_{i \in \mathbb{Z}}$ and $\left\{\gamma_{i}\right\}_{i \in \mathbb{Z}}$ satisfy the $(2,1)$ condition. 
Let $z(\cdot)=\left(z_{1}(\cdot), z_{2}(\cdot)\right)^{T}=\left(y(\cdot), y^{\prime}(\cdot)\right)^{T}$, we write the IDEPCA 37a $-37 \mathrm{~b}$ in the system form

$$
\begin{aligned}
z^{\prime}(t) & =\left(\begin{array}{cc}
0 & 1 \\
-4 & -3
\end{array}\right) z(t)+\left(\begin{array}{c}
0 \\
\kappa_{1} \sin (\pi t) z_{1}^{2}\left(2\left[\frac{t+1}{2}\right]\right)
\end{array}\right)+\left(\begin{array}{c}
0 \\
\kappa_{2} \cos (\pi t)-\kappa_{2} z_{1}^{2}(t) z_{2}(t)
\end{array}\right), \\
\left.\Delta z\right|_{t_{k}} & =\left(\begin{array}{cc}
a(t) & 0 \\
0 & b(t)
\end{array}\right) z\left(t_{k}^{-}\right)
\end{aligned}
$$

where

$$
A=\left(\begin{array}{rr}
0 & 1 \\
-4 & -3
\end{array}\right), \quad f\left(t, z(t), z\left(2\left[\frac{t+1}{2}\right]\right)\right)=\left(\begin{array}{l}
0 \\
\kappa_{1} \sin (\pi t) z_{1}^{2}\left(2\left[\frac{t+1}{2}\right]\right)
\end{array}\right)
$$

and

$$
g\left(t, z(t), z\left(2\left[\frac{t+1}{2}\right]\right)\right)=\left(\begin{array}{l}
0 \\
\kappa_{2} \cos (\pi t)-\kappa_{2} z_{1}^{2}(t) z_{2}(t)
\end{array}\right) .
$$

As all eigenvalues of the constant coefficient matrix A have negative real partes, then the linear homogeneous system $z^{\prime}(t)=A z(t)$ does not admit any nontrivial $\omega$ periodic solution, i.e., the condition $\left(\mathcal{N}_{\omega}\right)$ is satisfied. Let $\varphi(t)=\left(\varphi_{1}(t), \varphi_{2}(t)\right)^{T}$, $\psi(t)=\left(\psi_{1}(t), \psi_{2}(t)\right)^{T}$ and define $\mathbb{S}=\left\{z \in \mathbb{P}_{\omega},\|z\| \leq \mathcal{R}\right\}$, where $\mathcal{R} \in \mathbb{R}_{+}$satisfies the condition

$$
c_{G}\left(2 \mathcal{R} \kappa_{1}+2 \mathcal{R}^{2} \kappa_{2}+\kappa_{3}\right)<1
$$

where $\kappa_{3}=\max \left(\sup _{t \in[\tau, \tau+\omega]} a(t), \sup _{t \in[\tau, \tau+\omega]} b(t)\right)$.

Then, for $\varphi, \psi \in \mathbb{S}$ we have

$$
\begin{aligned}
& \|g(\cdot, \varphi(\cdot), \varphi(\gamma(\cdot)))-g(\cdot, \psi(\cdot), \psi(\gamma(\cdot)))\| \\
& \leq \sup _{t \in[\tau, \tau+\omega]}\left|\left(\begin{array}{l}
0 \\
\kappa_{2} \cos (\pi t)-\kappa_{2} \varphi_{1}^{2}(t) \varphi_{2}(t)
\end{array}\right)-\left(\begin{array}{l}
0 \\
\kappa_{2} \cos (\pi t)-\kappa_{2} \psi_{1}^{2}(t) \psi_{2}(t)
\end{array}\right)\right| \\
& \leq \sup _{t \in[\tau, \tau+\omega]}\left|\left(\kappa_{2}\left(\psi_{1}(t)+\varphi_{1}(t)\right) \varphi_{2}(t) \quad \kappa_{2} \psi_{1}^{2}(t)\right)\left(\begin{array}{c}
\psi_{1}(t)-\varphi_{1}(t) \\
\psi_{2}(t)-\varphi_{2}(t)
\end{array}\right)\right| \\
& \leq 2 \kappa_{2} \mathcal{R}^{2} \sup _{t \in[\tau, \tau+\omega]}\left|\left(\begin{array}{c}
\psi_{1}(t)-\varphi_{1}(t) \\
\psi_{2}(t)-\varphi_{2}(t)
\end{array}\right)\right|=2 \kappa_{2} \mathcal{R}^{2}\|\varphi-\psi\| \text {. }
\end{aligned}
$$

In a similar way, for $f$ we have

$$
\|f(\cdot, \varphi(\cdot), \varphi(\gamma(\cdot)))-f(\cdot, \psi(\cdot), \psi(\gamma(\cdot)))\| \leq 2 \kappa_{1} \mathcal{R}\|\varphi-\psi\|,
$$

for $J_{k}$ we obtain

$$
\left\|J_{k}\left(\varphi\left(t_{k}^{-}\right)\right)-J_{k}\left(\psi\left(t_{k}^{-}\right)\right)\right\| \leq \kappa_{3}\|\varphi-\psi\| .
$$

By Theorem 15, the IDEPCA (37a)-(37b) has a unique 2-periodic solution in $\mathbb{S}$.

Acknowledgments The author thanks the referees very much for their valuable suggestions which made this paper much improved. This research was in part supported by PGI 03-2020 DIUMCE. 


\section{REFERENCES}

[1] Akhmet, M. U., Nonlinear hybrid continuous/discrete-time models, Atlantis Press, Paris, 2011.

[2] Alwan, M. S., Liu, X., Stability of singularly perturbed switched systems with time delay and impulsive effects, Nonlinear Analysis: TMA., 71 (2009), 4297-4308.

[3] Bereketoglu, H., Seyhan, G., Ogun, A., Advanced impulsive differential equations with piecewise constant arguments, Mathematical Modelling and Analysis, 15 (2) (2010) 175-187.

[4] Burton, T. A., A fixed-point theorem of Krasnoselskii, Appl. Math. Lett., 11 (1998), 85-88.

[5] Busenberg, S., Cooke, K. L., Models of vertically transmitted diseases with sequentialcontinuous dynamics, in: V. Lakshmikantham (Ed.), Nonlinear Phenomena in Mathematical Sciences, Academic Press, New York, 1982, 179-187.

[6] Cabada, A., Ferreiro, J. B., Nieto, J. J., Green's function and comparison principles for first order periodic differential equations with piecewise constant arguments, J. Math. Anal. Appl., 291 (2004), 690-697.

[7] Castillo, S., Pinto, M., Torres, R., Asymptotic formulae for solutions to impulsive differential equations with piecewise constant argument of generalized type, Electron. J. Differential Equations, Vol. 2019 (2019), No. 40, pp. 1-22.

[8] Cooke, K. L., Wiener, J., An equation alternately of retarded and advanced type, Proc. Amer. Math. Soc., 99 (1987), 726-732.

[9] Chiu K.-S., Pinto, M., Periodic solutions of differential equations with a general piecewise constant argument and applications, E. J. Qualitative Theory of Diff. Equ., 46 (2010), 1-19.

[10] Chiu, K.-S., Stability of oscillatory solutions of differential equations with a general piecewise constant argument, E. J. Qualitative Theory of Diff. Equ., 88 (2011), 1-15.

[11] Chiu, K.-S., Periodic solutions for nonlinear integro-differential systems with piecewise constant argument, The Scientific World Journal, vol. 2014 (2014), Article ID 514854, 14 pages.

[12] Chiu, K.-S., Existence and global exponential stability of equilibrium for impulsive cellular neural network models with piecewise alternately advanced and retarded argument, Abstract and Applied Analysis, vol. 2013 (2013),Article ID 196139, 13 pages.

[13] Chiu, K.-S., Pinto, M., Jeng, J.-C., Existence and global convergence of periodic solutions in recurrent neural network models with a general piecewise alternately advanced and retarded argument, Acta Appl. Math., 133 (2014), 133-152.

[14] Chiu, K.-S., Pinto, M., Oscillatory and periodic solutions in alternately advanced and delayed differential equations, Carpathian J. Math., 29 (2) (2013), 149-158.

[15] Chiu, K.-S., On generalized impulsive piecewise constant delay differential equations, Science China Mathematics, 58 (2015), 1981-2002.

[16] Chiu, K.-S., Exponential stability and periodic solutions of impulsive neural network models with piecewise constant argument, Acta Appl. Math., 151 (2017), 199-226.

[17] Chiu, K.-S., Asymptotic equivalence of alternately advanced and delayed differential systems with piecewise constant generalized arguments, Acta Math. Sci., 38 (2018), 220-236.

[18] Chiu, K.-S., Li, T., Oscillatory and periodic solutions of differential equations with piecewise constant generalized mixed arguments, Math. Nachr., 292 (2019), 2153-2164.

[19] Chiu, K.-S., Green's function for periodic solutions in alternately advanced and delayed differential systems, Acta Math. Appl. Sin. Engl. Ser. 36 (4) (2020), 936-951.

[20] Jayasree, K. N., Deo, S. G., On piecewise constant delay differential equations, J. Math. Anal. Appl., 169 (1992), 55-69.

[21] Karakoc, F., Bereketoglu, H., Seyhan, G., Oscillatory and periodic solutions of impulsive differential equations with piecewise constant argument, Acta Appl. Math., 110(1) (2009), $499-510$ 
[22] Karakoc, F., Bereketoglu, H., Some results for linear impulsive delay differential equations, Dynamics of Cont. Discrete and Impulsive Systems Series A: Mathematical Analysis, 16 (2009), 313-326.

[23] Karakoc, F., Ogun Unal, A., Bereketoglu,H., Oscillation of nonlinear differential equations with piecewise constant arguments. E. J. Qualitative Theory of Diff. Equ., 49 (2013), 1-12.

[24] Li, X., Weng, P., Impulsive stabilization of two kinds of second-order linear delay differential equations, J. Math. Anal. Appl., 291 (2004), 270-281.

[25] Martynyuk, A. A., Shen, J. H., Stavroulakis, I. P., Stability theorems in impulsive functional differential equations with infinite delay, Advances in stability theory at the end of the 20th century, Stability Control Theory Methods Appl., 13 (2003), 153-174.

[26] Lakshmikantham, V., Bainov, D. D., Simeonov, P. S., Theory of Impulsive Differential Equations, World Scientific, Singapore, 1989.

[27] Myshkis, A.D., On certain problems in the theory of differential equations with deviating arguments, Uspekhi Mat. Nauk., 32 (1977), 173-202.

[28] Nieto, J. J. and, Rodríguez-López, R., Green's function for second-order periodic boundary value problems with piecewise constant arguments, J. Math. Anal. Appl., 304 (2005), 33-57.

[29] Oztepe, G. S., Karakoc, F., Bereketoglu, H., Oscillation and periodicity of a second order impulsive delay differential equation with a piecewise constant argument, Communications in Mathematics, 25 (2017), 89-98.

[30] Pinto, M., Cauchy and Green matrices type and stability in alternately advanced and delayed differential systems, J. Difference Equ. Appl., 17 (2) (2011), 235-254.

[31] Samoilenko, A. M., Perestyuk, N. A., Impulsive Differential Equations, World Scientific Singapore, 1995.

[32] Raffoul, Y. N., Periodic solutions for neutral nonlinear differential equations with functional delay, Electron. J. Differential Equations, Vol. 2003 (2003), no. 102, 1-7.

[33] Shah, S. M., Wiener, J., Advanced differential equations with piecewise constant argument deviations, Internat, J. Math. and Math. Sci., 6 (1983), 671-703.

[34] Smart, D. R., Fixed Points Theorems, Cambridge University Press, Cambridge, 1980.

[35] Wiener, J., Generalized Solutions of Functional Differential Equations, World Scientific, Singapore, 1993.

[36] Wang, G.-Q., Cheng, S.-S., Existence and uniqueness of periodic solutions for a second-order nonlinear differential equation with piecewise constant argument, Int. J. Math. Math. Sci., 2009, Art. ID 950797, 14 pp.

[37] Wiener, J., Lakshmikantham, V., Differential equations with piecewise constant argument and impulsive equations, Nonlinear Stud., 7 (2000), 60-69.

[38] Xia, Y. H., Huang, Z., Han, M., Existence of almost periodic solutions for forced perturbed systems with piecewise constant argument, J. Math. Anal. Appl., 333 (2007), 798-816. 